\title{
The Expected Fitness Cost of a Mutation Fixation under the One-dimensional Fisher Model
}

\author{
Liqing Zhang ${ }^{1, *}$ and Layne T. Watson ${ }^{2}$ \\ ${ }^{1}$ Department of Computer Science, \\ Virginia Polytechnic Institute \& State University, \\ Blacksburg, VA 24061 \\ ${ }^{2}$ Departments of Computer Science and Mathematics, \\ Virginia Polytechnic Institute \& State University \\ Blacksburg, VA 24061 \\ *To whom correspondence should be addressed; \\ E-mail: lqzhang@vt.edu
}

Phone: 540-231-9413. Fax: 540-231-6075 


\begin{abstract}
This paper employs Fisher's model of adaptation to understand the expected fitness effect of fixing a mutation in a natural population. Fisher's model in one dimension admits a closed form solution for this expected fitness effect. A combination of different parameters, including the distribution of mutation lengths, population sizes, and the initial state that the population is in, are examined to see how they affect the expected fitness effect of state transitions. The results show that the expected fitness change due to the fixation of a mutation is always positive, regardless of the distributional shapes of mutation lengths, effective population sizes, and the initial state that the population is in. The further away the initial state of a population is from the optimal state, the slower the population returns to the optimal state. Effective population size (except when very small) has little effect on the expected fitness change due to mutation fixation. The always positive expected fitness change suggests that small populations may not necessarily be doomed due to the runaway process of fixation of deleterious mutations.
\end{abstract}

Key words: Fisher's model, effective population size, compensatory mutation, generalized Riemann zeta function, incomplete gamma function. 


\section{INTRODUCTION}

The statistician R. Fisher [4] proposed a geometrical model to understand the nature of adaptation. The basic idea of his model can be illustrated using a simple one-dimensional system. Imagine that a trait has the optimal state at the origin, the population's current state can be represented by point $A$ on the real coordinate line, and the distance between point $A$ and the origin $O$ represents the fitness of the population at state $A$. Mutations can occur with both magnitude and direction, which will drive the population either further away from the population optimum point $O$, or towards the optimum point $O$. One can therefore model the dynamics of mutations by tracking the movement of the population states owing to the fixation of mutations.

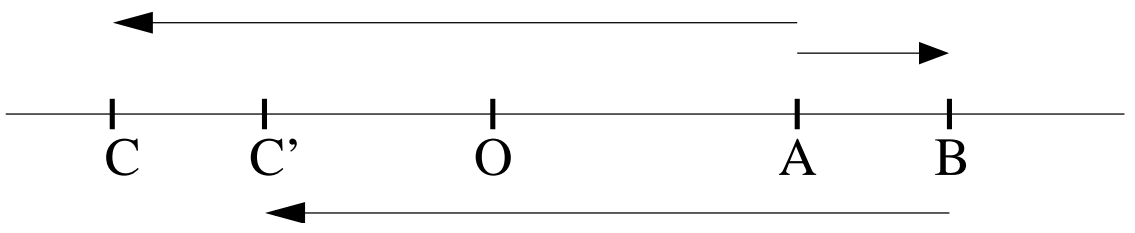

Figure 1: Fisher's model of adaptation in one dimension

The attractiveness of Fisher's model lies in the fact that it nicely incorporates the nonindependent nature of multiple mutations. For example, in the one-dimensional system, suppose that the population starts at state $A$, that is, all the individuals in the population carry the allele A. A mutation of a certain type will take the population to state $B$, where all the individuals in the population carry the mutated type $B$. Similarly, from state $A$ a mutation of a different type will take the population to state $C$, where all the individuals carry the mutant type $C$. Compared with the original state $A$, both mutations are deleterious and move the population to states $(B$ or $C$ ) that have lower fitness than the original state $A$. However, if both mutants appear and get fixed together, the population will have a fitness gain at state $C^{\prime}$ from the original state $A$. Therefore, both mutations are deleterious and reduce the population fitness when fixed individually. However, the joint fixation of the two leads to a fitness gain instead - the two deleterious mutations are compensatory. Therefore, Fisher's model has built-in nonindependence, 
and elegantly models the nonindependent feature of mutations. Fisher's model of adaptation has been applied to study compensatory mutations by, e.g., Poon and Otto [9], who studied the effect of compensatory mutations with respect to the number of character dimensions. They concluded that the effects of compensatory mutations become more pronounced when the number of character dimensions increases.

This paper examines the expected fitness cost of transition from one population state to another, using Fisher's model in one dimension, where closed form analytic solutions exist. In $n>1$ dimensions, analytic solutions do not exist if the distance measure is the 2-norm (Euclidean distance). The analytic techniques employed here for $n=1$ can be extended to $n>1$ if the 1-norm is used for distance, but then each two cases (based on a sign) for $n=1$ become $2^{n}$ cases, making the closed form expressions completely unwieldy. Note that the one-dimensional model considered here is not so restrictive as it might appear. It has been shown that the $n$-dimensional Fisher model can be reduced to two dimensions (polar coordinates), for which the marginal distributions are one-dimensional $[4,6,8]$. Thus the one-dimensional results here apply to the marginal distributions for the general case ( $n$ dimensions reduced to two), and are of some interest. Assuming a gamma probability distribution for the mutation magnitude, the present work derives analytically the mean fitness cost of a transition, and studies the effect of a variety of parameters, including the population size and different initial states, on the next state transition. The biological implications of the findings are discussed.

\section{MATHEMATICAL DERIVATIONS}

This section focuses on deriving the expected fitness effect of mutations moving the population from one state to another. Because the comparison is between the current population state and the next state, the fitness effect is thus the comparison of these two states. Assume that the distance away from the optimum point (origin on real line) corresponds to the fitness $w$ of the

state via the equations $w(A)=e^{-|z|}$ and $w(B)=e^{-\left|z^{\prime}\right|}$, where $z$ and $z^{\prime}$ are signed real numbers, 
representing the coordinate positions of population states $A$ and $B$. Note that this definition of $w$ is similar to that of Waxman and Welch [13]. The standard definition [9] of the selection coefficient of mutation from $A$ to $B$ is

$$
s=\frac{W(B)}{W(A)}-1=\frac{e^{-\left|z^{\prime}\right|}-e^{-|z|}}{e^{-|z|}}=e^{|z|-\left|z^{\prime}\right|}-1 \approx 1-\left|\frac{z^{\prime}}{z}\right|
$$

for $|z| \approx\left|z^{\prime}\right|$ and $|z| \approx 1$. The first assumption, $|z| \approx\left|z^{\prime}\right|$, corresponds to $|s| \approx 0$, a common assumption in the literature (that $|s|$ is large with vanishingly small probability). The second assumption, $|z| \approx 1$ for the current population state, corresponds to scaling the distance measure $z$. It turns out that for $s=1-\left|\frac{z^{\prime}}{z}\right|$ closed form expressions can be derived and that is done below. However, all the numerical results are for the standard $s=W(B) / W(A)-1$, with the integrals done numerically (with Mathematica).

Due to the uncertainty about the distribution of mutations, assume that mutation magnitude from one state to another (i.e., $\left.\left|z^{\prime}-z\right|\right)$ is gamma distributed, which incorporates a variety of distribution shapes (with different parameters) and thus models a rich collection of mutation scenarios. Specifically, let the probability density function of mutation to $z^{\prime}$ from $z$ be

$$
f\left(z^{\prime}\right)=\frac{\left|z^{\prime}-z\right|^{\alpha-1} \beta^{\alpha} e^{-\beta\left|z^{\prime}-z\right|}}{\Gamma(\alpha)}
$$

where $\alpha$ and $\beta$ are the shape and location parameters in the gamma distribution. The fixation probability $u(s)$ of the mutation state has been given by Crow and Kimura [2] as

$$
u(s)=\frac{1-e^{-2 N_{e} s / N}}{1-e^{-4 N_{e} s}}
$$

where $N_{e}$ is the effective population size, and $N$ is the population size. For simplicity, the analysis here takes $N_{e}=N$.

Following Fisher's geometrical model of adaptation, assume that the mutation magnitude 
can be used to define the relative log fitness change

$$
\frac{\log w(B)-\log w(A)}{-\log w(A)}=\frac{-\left|z^{\prime}\right|-(-|z|)}{|z|}=1-\left|\frac{z^{\prime}}{z}\right|
$$

of the mutation away from the optimal state, where (for the analytical derivation) the selection coefficient $s=1-\left|\frac{z^{\prime}}{z}\right|$, for a transition from state $z$ to state $z^{\prime}$. Assuming that the magnitude of mutations has a gamma distribution, and the fitness effect of a new mutation depends on the current state of the population mutation, then the gamma probability density function times the fixation probability of the mutation times the fitness change $s$ (for diploid populations, $2 s$ is used), integrated over all new states $z^{\prime}$, yields the expected (relative) fitness effect of a state transition from $z$ :

$$
\begin{aligned}
W(z) & =\int_{-\infty}^{\infty} s f\left(z^{\prime}\right) u(s) d z^{\prime} \\
& =\int_{-\infty}^{\infty}\left(1-\left|\frac{z^{\prime}}{z}\right|\right) \frac{\left|z^{\prime}-z\right|^{\alpha-1} \beta^{\alpha} e^{-\beta\left|z^{\prime}-z\right|}}{\Gamma(\alpha)} \frac{1-e^{-2\left(1-\left|\frac{z^{\prime}}{z}\right|\right)}}{1-e^{-4 N_{e}\left(1-\left|\frac{z^{\prime}}{z}\right|\right)}} d z^{\prime}
\end{aligned}
$$

where $\alpha>0, \beta>0$, and $N_{e}>0$. It is clear that $W(z)$ reflects the dependency of fitness effect on the relative location $\left|z^{\prime}-z\right|$ of the new mutation and the current mutation location $z$. Decompose $W(z)$ as $W(z)=W_{1}(z)-W_{2}(z)$ where

$$
W_{1}(z)=\int_{-\infty}^{\infty} f\left(z^{\prime}\right) u(s) d z^{\prime}, \quad W_{2}(z)=\int_{-\infty}^{\infty}\left|\frac{z^{\prime}}{z}\right| f\left(z^{\prime}\right) u(s) d z^{\prime}
$$

These integrals $W_{1}$ and $W_{2}$ will be expressed in terms of the gamma function $\Gamma(\alpha)=\int_{0}^{\infty} x^{\alpha-1}$ $e^{-x} d x$, the incomplete gamma function $\gamma(\alpha, y)=\int_{y}^{\infty} x^{\alpha-1} e^{-x} d x$, the generalized incomplete gamma function $\hat{\gamma}(\alpha, x, y)=\gamma(\alpha, x)-\gamma(\alpha, y)$, and the generalized Riemann zeta function $\mathcal{Z}(s, a)=\sum_{k=0}^{\infty} \frac{1}{(k+a)^{s}}$.

Because of the absolute values, doing the integrals analytically requires considering different cases. First, consider the case when $z>0$. Write the first part of the integral in equation (4) as 
$W_{1}(z)=D_{1}+D_{2}+D_{3}$, where

$$
\begin{aligned}
& D_{1}=\int_{-\infty}^{0} \frac{\left(z-z^{\prime}\right)^{\alpha-1} \beta^{\alpha} e^{-\beta\left(z-z^{\prime}\right)}}{\Gamma(\alpha)} \frac{1-e^{-2\left(1+\frac{z^{\prime}}{z}\right)}}{1-e^{-4 N_{e}\left(1+\frac{z^{\prime}}{z}\right)}} d z^{\prime}, \\
& D_{2}=\int_{0}^{z} \frac{\left(z-z^{\prime}\right)^{\alpha-1} \beta^{\alpha} e^{-\beta\left(z-z^{\prime}\right)}}{\Gamma(\alpha)} \frac{1-e^{-2\left(1-\frac{z^{\prime}}{z}\right)}}{1-e^{-4 N_{e}\left(1-\frac{z^{\prime}}{z}\right)}} d z^{\prime}, \\
& D_{3}=\int_{z}^{\infty} \frac{\left(z^{\prime}-z\right)^{\alpha-1} \beta^{\alpha} e^{-\beta\left(z^{\prime}-z\right)}}{\Gamma(\alpha)} \frac{1-e^{-2\left(1-\frac{z^{\prime}}{z}\right)}}{1-e^{-4 N_{e}\left(1-\frac{z^{\prime}}{z}\right)}} d z^{\prime} .
\end{aligned}
$$

Second, consider the case when $z<0$, and write the first part of the integral in equation (4) as $W_{1}(z)=D_{4}+D_{5}+D_{6}$, where

$$
\begin{aligned}
& D_{4}=\int_{0}^{\infty} \frac{\left(z^{\prime}-z\right)^{\alpha-1} \beta^{\alpha} e^{-\beta\left(z^{\prime}-z\right)}}{\Gamma(\alpha)} \frac{1-e^{-2\left(1+\frac{z^{\prime}}{z}\right)}}{1-e^{-4 N_{e}\left(1+\frac{z^{\prime}}{z}\right)}} d z^{\prime}, \\
& D_{5}=\int_{-\infty}^{z} \frac{\left(z-z^{\prime}\right)^{\alpha-1} \beta^{\alpha} e^{-\beta\left(z-z^{\prime}\right)}}{\Gamma(\alpha)} \frac{1-e^{-2\left(1-\frac{z^{\prime}}{z}\right)}}{1-e^{-4 N_{e}\left(1-\frac{z^{\prime}}{z}\right)}} d z^{\prime}, \\
& D_{6}=\int_{z}^{0} \frac{\left(z^{\prime}-z\right)^{\alpha-1} \beta^{\alpha} e^{-\beta\left(z^{\prime}-z\right)}}{\Gamma(\alpha)} \frac{1-e^{-2\left(1-\frac{z^{\prime}}{z}\right)}}{1-e^{-4 N_{e}\left(1-\frac{z^{\prime}}{z}\right)}} d z^{\prime} .
\end{aligned}
$$

A closed form expression for each of $D_{1}, D_{2}, \ldots, D_{6}$ is derived in the appendix.

Given $W_{1}(z), W_{2}(z)=\int_{-\infty}^{\infty}\left|\frac{z^{\prime}}{z}\right| f\left(z^{\prime}\right) u(s) d z^{\prime}$ is straightforward to compute. As before, similar to $W_{1}(z)$, write $W_{2}(z)=D_{1}^{\prime}+D_{2}^{\prime}+D_{3}^{\prime}$ for $z>0$ and $W_{2}(z)=D_{4}^{\prime}+D_{5}^{\prime}+D_{6}^{\prime}$ for $z<0$, where

$$
\begin{aligned}
& D_{1}^{\prime}=\int_{-\infty}^{0} \frac{\frac{-z^{\prime}}{z}\left(z-z^{\prime}\right)^{\alpha-1} \beta^{\alpha} e^{-\beta\left(z-z^{\prime}\right)}}{\Gamma(\alpha)} \frac{1-e^{-2\left(1+\frac{z^{\prime}}{z}\right)}}{1-e^{-4 N_{e}\left(1+\frac{z^{\prime}}{z}\right)}} d z^{\prime}, \\
& D_{2}^{\prime}=\int_{0}^{z} \frac{\frac{z^{\prime}}{z}\left(z-z^{\prime}\right)^{\alpha-1} \beta^{\alpha} e^{-\beta\left(z-z^{\prime}\right)}}{\Gamma(\alpha)} \frac{1-e^{-2\left(1-\frac{z^{\prime}}{z}\right)}}{1-e^{-4 N_{e}\left(1-\frac{z^{\prime}}{z}\right)}} d z^{\prime}, \\
& D_{3}^{\prime}=\int_{z}^{\infty} \frac{\frac{z^{\prime}}{z}\left(z^{\prime}-z\right)^{\alpha-1} \beta^{\alpha} e^{-\beta\left(z^{\prime}-z\right)}}{\Gamma(\alpha)} \frac{1-e^{-2\left(1-\frac{z^{\prime}}{z}\right)}}{1-e^{-4 N_{e}\left(1-\frac{z^{\prime}}{z}\right)}} d z^{\prime}, \\
& D_{4}^{\prime}=\int_{0}^{\infty} \frac{\frac{-z^{\prime}}{z}\left(z^{\prime}-z\right)^{\alpha-1} \beta^{\alpha} e^{-\beta\left(z^{\prime}-z\right)}}{\Gamma(\alpha)} \frac{1-e^{-2\left(1+\frac{z^{\prime}}{z}\right)}}{1-e^{-4 N_{e}\left(1+\frac{z^{\prime}}{z}\right)}} d z^{\prime}, \\
& D_{5}^{\prime}=\int_{-\infty}^{z} \frac{\frac{z^{\prime}}{z}\left(z-z^{\prime}\right)^{\alpha-1} \beta^{\alpha} e^{-\beta\left(z-z^{\prime}\right)}}{\Gamma(\alpha)} \frac{1-e^{-2\left(1-\frac{z^{\prime}}{z}\right)}}{1-e^{-4 N_{e}\left(1-\frac{z^{\prime}}{z}\right)}} d z^{\prime},
\end{aligned}
$$




$$
D_{6}^{\prime}=\int_{z}^{0} \frac{\frac{z^{\prime}}{z}\left(z^{\prime}-z\right)^{\alpha-1} \beta^{\alpha} e^{-\beta\left(z^{\prime}-z\right)}}{\Gamma(\alpha)} \frac{1-e^{-2\left(1-\frac{z^{\prime}}{z}\right)}}{1-e^{-4 N_{e}\left(1-\frac{z^{\prime}}{z}\right)}} d z^{\prime} .
$$

Then $D_{1}^{\prime}$ can be rewritten as

$$
\begin{aligned}
D_{1}^{\prime} & =\frac{1}{z}\left[\int_{-\infty}^{0} \frac{\left(z-z^{\prime}\right)\left(z-z^{\prime}\right)^{\alpha-1} \beta^{\alpha} e^{-\beta\left(z-z^{\prime}\right)}}{\Gamma(\alpha)} \frac{1-e^{-2\left(1+\frac{z^{\prime}}{z}\right)}}{1-e^{-4 N_{e}\left(1+\frac{z^{\prime}}{z}\right)}} d z^{\prime}\right. \\
& \left.-\int_{-\infty}^{0} \frac{z\left(z-z^{\prime}\right)^{\alpha-1} \beta^{\alpha} e^{-\beta\left(z-z^{\prime}\right)}}{\Gamma(\alpha)} \frac{1-e^{-2\left(1+\frac{z^{\prime}}{z}\right)}}{1-e^{-4 N_{e}\left(1+\frac{z^{\prime}}{z}\right)}} d z^{\prime}\right] \\
& =\frac{1}{z} \int_{-\infty}^{0} \frac{\left(z-z^{\prime}\right)^{\alpha} \beta^{\alpha} e^{-\beta\left(z-z^{\prime}\right)}}{\Gamma(\alpha)} \frac{1-e^{-2\left(1+\frac{z^{\prime}}{z}\right)}}{1-e^{-4 N_{e}\left(1+\frac{z^{\prime}}{z}\right)}} d z^{\prime}-D_{1} \\
& =\frac{1}{z} D_{1, \alpha}-D_{1} .
\end{aligned}
$$

Notice that the integral $D_{1, \alpha}$ has the same integrand as $D_{1}$ except for an extra factor of $\left|z^{\prime}-z\right|$. The effect of this is to replace every occurrence of $\alpha$ by $\alpha+1$ in the final integral formula for $D_{1, \alpha}$, except for the factor $\beta^{\alpha} / \Gamma(\alpha)$, which remains unchanged. This same pattern holds for all the $D_{i}^{\prime}$, precisely,

$$
\begin{aligned}
D_{1}^{\prime} & =\frac{1}{z} D_{1, \alpha}-D_{1}, \\
D_{2}^{\prime} & =-\frac{1}{z} D_{2, \alpha}+D_{2}, \\
D_{3}^{\prime} & =\frac{1}{z} D_{3, \alpha}+D_{3}, \\
D_{4}^{\prime} & =-\frac{1}{z} D_{4, \alpha}-D_{4}, \\
D_{5}^{\prime} & =-\frac{1}{z} D_{5, \alpha}+D_{5}, \\
D_{6}^{\prime} & =\frac{1}{z} D_{6, \alpha}+D_{6},
\end{aligned}
$$

where the final integral formula for $D_{i, \alpha}$ differs from that for $D_{i}$ as just described for $D_{1, \alpha}$ and $D_{1}$

Finally, for $z>0$,

$$
W(z)=W_{1}(z)-W_{2}(z)
$$




$$
\begin{aligned}
& =D_{1}+D_{2}+D_{3}-\left(\frac{1}{z} D_{1, \alpha}-D_{1}\right)-\left(-\frac{1}{z} D_{2, \alpha}+D_{2}\right)-\left(\frac{1}{z} D_{3, \alpha}+D_{3}\right) \\
& =2 D_{1}-\frac{1}{z} D_{1, \alpha}+\frac{1}{z} D_{2, \alpha}-\frac{1}{z} D_{3, \alpha},
\end{aligned}
$$

and for $z<0$,

$$
\begin{aligned}
W(z) & =W_{1}(z)-W_{2}(z) \\
& =D_{4}+D_{5}+D_{6}-\left(-\frac{1}{z} D_{4, \alpha}-D_{4}\right)-\left(-\frac{1}{z} D_{5, \alpha}+D_{5}\right)-\left(\frac{1}{z} D_{6, \alpha}+D_{6}\right) \\
& =2 D_{4}+\frac{1}{z} D_{4, \alpha}+\frac{1}{z} D_{5, \alpha}-\frac{1}{z} D_{6, \alpha} .
\end{aligned}
$$

\section{RESULTS AND DISCUSSION}

\section{The effect of the distribution of mutation lengths}

The distribution of mutation lengths in nature is unknown. However, because the gamma distribution can represent a variety of distribution shapes, employing it for the analysis covers many plausible approximations for the true distribution. In order to examine the effect of different distributions for mutation lengths, $W(z)$ is computed for different shapes to examine the effect of distributional shapes on the expected fitness changes. The simplest form is exponential, which has been used previously to approximate the distribution of the fitness effect of deleterious mutations (e.g., [15]) and rare beneficial mutations [8]. Consider first the exponential distribution, where $\alpha=1$, and $\beta$ ranges within $(0,10]$. Shown in Figure 2 , for exponential distributions with different decay rates $\beta>0$, the expected fitness effect of the fixation of a new mutation is always positive, suggesting that while mutations can take the population either to a state with lower fitness than the current one or a state with higher fitness, the mean fitness change will be a gain rather than a loss. In particular, for small $\beta$ near zero, the expected fitness gain from the current state $z=4$ increases with $\beta$, peaks around $\beta=0.286$ (the expected fitness effect reaches the maximum of 0.277 ) and then decreases as $\beta$ increases; past the peak, the larger $\beta$ is, the smaller the effect of the fixation of a new mutation on the fitness change of the population. This 
observation is easy to understand because a large $\beta$ value means that most of the mutations have a very small mutation length from the current state of the population, therefore, the fixation of the new mutation is expected to have a small effect on the population fitness change. For very small $\beta \approx 0$, both large and small mutation lengths occur with high probability, and since small mutation lengths tend to be beneficial and large mutation lengths tend to be deleterious, the effect of deleterious mutations nearly balances out the effect of beneficial mutations $(W(z) \approx 0)$. Increasing $\beta$ gives the smaller beneficial mutation lengths an edge, so $W(z)$ increases rapidly, until it peaks at the crossover point in the gain/loss ratio for small length mutations. This crossover occurs at the switch between prevalence of long length mutations (small $\beta$ ) and prevalence of short length mutations (large $\beta$ ).

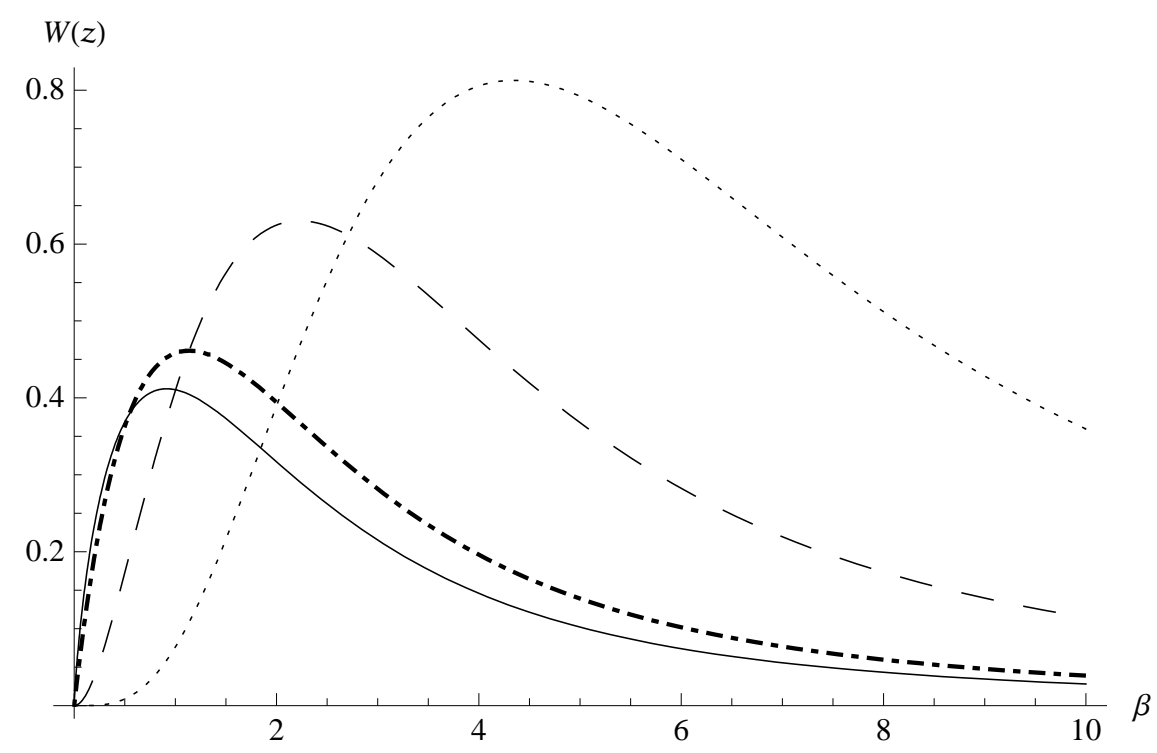

Figure 2: The effect of the distributional shapes of mutation lengths on the expected fitness change of a new mutation with $z=4$ and $N_{e}=1000$ for all the curves, but with different $\alpha: \alpha=0.8$ (black), $\alpha=1$ (dotdashed), $\alpha=2$ (dashed), and $\alpha=4$ (dotted).

Figure 2 also shows the effect of the distribution of mutation lengths for different $\alpha$ s. For small $\beta \approx 0$, the expected fitness gain due to fixation of a new mutation tends to be lower for larger $\alpha$, while for $\beta \gg 1$, tends to be higher for larger $\alpha$. With the current parameter settings, for example, when $\beta=2$, the expected fitness gain is much larger for large $\alpha$ than for small $\alpha$. 
In general, larger $\alpha$ s tend to have a wider range of $\beta$ within which the expected fitness gains are large owing to the fixation of a new mutation than smaller $\alpha$ s. Moreover, for all different values of $\alpha$ and $\beta$, fixing one, there is always a maximum expected fitness gain with respect to the

other, which can be obtained by setting the partial derivatives $\frac{\partial W}{\partial \alpha}$ or $\frac{\partial W}{\partial \beta}$ to zero and solving for $\alpha$ or $\beta$.

Previous studies have shown that large coefficients of variation in the fitness effect of both deleterious and beneficial mutations enable small populations to persist [17, 18]. This effect is explored here by varying the coefficient of variation of mutation lengths to see what effect it has on the expected fitness change of a population. Since the coefficient of variation of a gamma distribution (with shape parameter $\alpha$, scale parameter $\beta$, mean $\alpha / \beta$, variance $\alpha / \beta^{2}$ ) is equal to $1 / \sqrt{\alpha}$, consider the relationship between $W(z)$ and $\alpha$ for different initial states (i.e., different $z$ ) with the same scale factor $\beta$, shown in Figure 3. Interestingly, for a specific initial state (e.g., $z=4$ ), the expected fitness gain increases with $\alpha$, reaches a maximum, and then approaches zero asymptotically as $\alpha \rightarrow \infty$. This shows that under the Fisher geometric adaptation model, the expected fitness gain is not a simple linear (or even monotone) function of the coefficient of variation of mutation lengths; since $\alpha \rightarrow 0$ implies the coefficient of variation $1 / \sqrt{\alpha} \rightarrow \infty$, a larger coefficient of variation for mutation lengths does not necessarily lead to higher expected fitness gains. Given the definition of fitness effect $s=\frac{|z|-\left|z^{\prime}\right|}{|z|}$, there might appear to be a strong correlation between the coefficient of variation of $s$ and that of the mutation length $\left|z^{\prime}-z\right|$, but the above observation indicates otherwise. Biologically, it is tempting to think that the coefficient of variation for mutation lengths should be positively correlated with the coefficient of variation for fitness effect, but one can imagine the counter-effect can also happen.

\section{The effect of the initial state}

Consider next the effect on $W(z)$ of changing the initial state $z$. Figure 4 shows that the starting state does affect the expected relative fitness change due to the fixation of a mutation. 


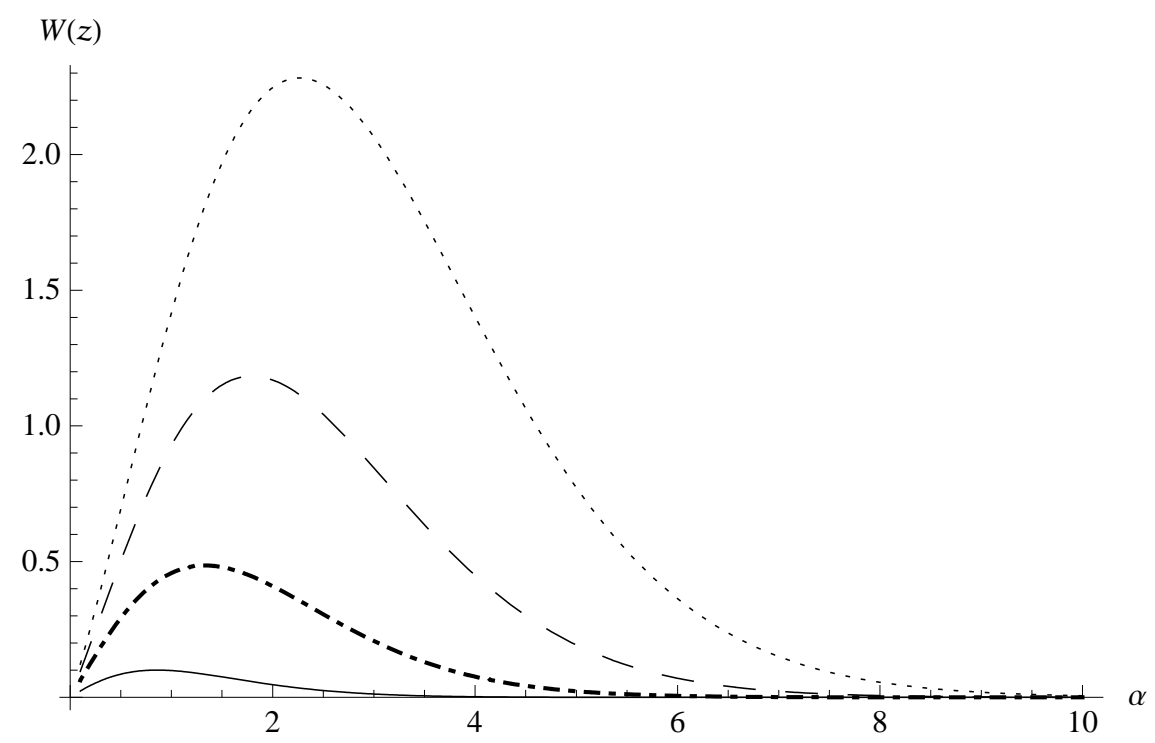

Figure 3: The effect of the distributional shapes of mutation lengths on the expected fitness change of a new mutation with $\beta=1$ and $N_{e}=100$ for all the curves, but with different initial states: $z=1$ (black), $z=4$ (dotdashed), $z=8$ (dashed), and $z=10$ (dotted).

For the same distribution of mutation lengths, the expected fitness gain for mutation fixation increases with the distance from the "optimal" state (the origin), and approaches a constant asymptotically as $|z| \rightarrow \infty$. The asymptotic value of $W(z)$ decreases with increasing $\beta$. It was discovered, though not shown in Figure 4, that when $\beta \leq 1$, the expected fitness gain $W(z) \rightarrow \infty$ as $z \rightarrow \infty$. This can be seen mathematically by noting that the maximum of the integrand in (4) occurs at $z^{\prime}=0$, where the exponential term $e^{|z|}$ overwhelms the exponential term $e^{-\beta|z|}$, causing the integrand (and integral) to increase without limit as $z \rightarrow \infty$. This has no biological interpretation, since distributions with $\beta<1$ correspond to large mutation lengths $\left|z^{\prime}-z\right|$ occurring with probability $\gg 0$, which is generally not true biologically.

\section{The effect of effective population sizes}

Population size and especially the effective population size is an important parameter in various evolutionary models, and plays an important role in determining the evolutionary trajectory of small populations and in determining the evolutionary fates of newly arising mutations. The 


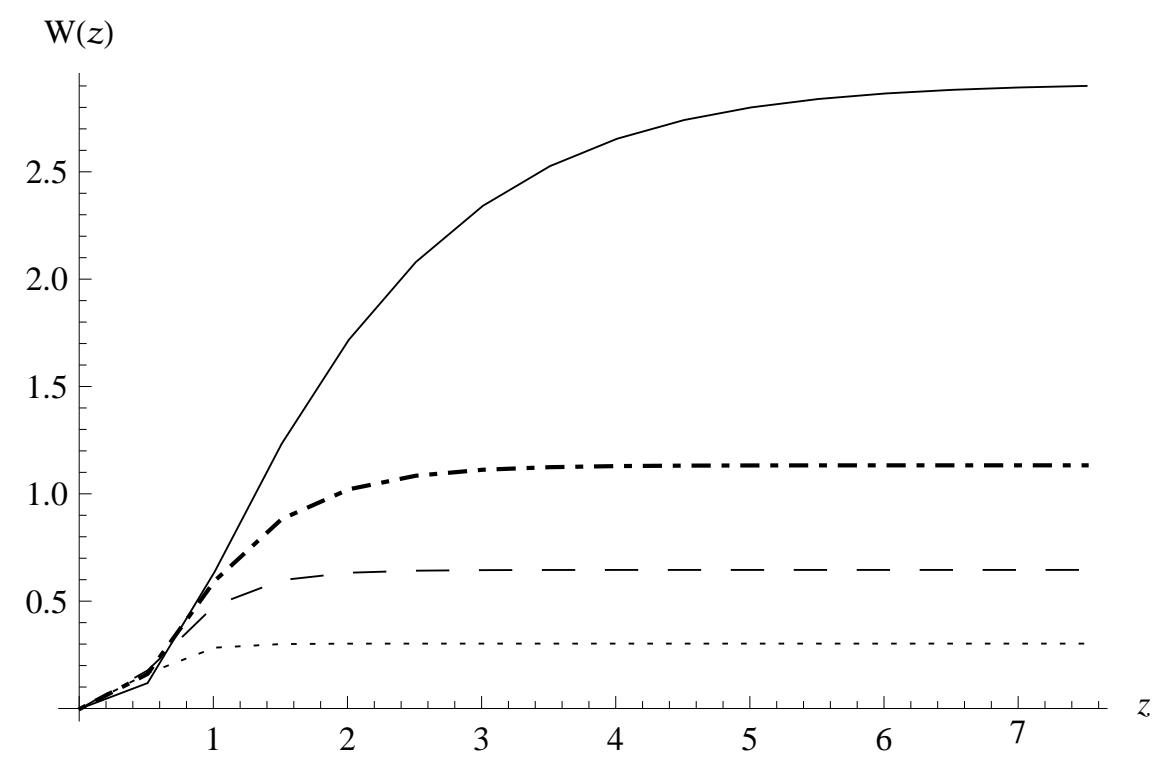

Figure 4: The effect of the initial state on the expected fitness change of a new mutation with $\alpha=2$ and $N_{e}=100$ for all the curves, but with different $\beta: \beta=2$ (black), $\beta=3$ (dotdashed), $\beta=4$ (dashed), and $\beta=6$ (dotted).

effective population sizes of various species in nature can be difficult to measure. The mathematical derivations earlier were simplified by assuming that $N_{e}=N$. However, existing studies show, in several species surveyed, the effective population size $\left(N_{e}\right)$ is usually much less than the census population size $(N)$, with an estimated fraction of $N_{e}=0.1 N$. The derivation for $N_{e} \neq N$ of the analytic expression for $W(z)$ follows along the lines of the derivation for $N_{e}=N$ and is omitted here.

Consider the effect of $N_{e}$ on the expected fitness change from one population state to another. From (3), it is clear that changing the effective population size $N_{e}$ should have little effect on the expected fitness change due to the fixation of a new mutation, since unless $N_{e}$ is really small, $u(s) \approx 1-e^{-2 s N_{e} / N}$. Changing the ratio $\frac{N_{e}}{N}$ has only a small effect on the final results for $\frac{N_{e}}{N}>1$ (Figure 5). Therefore, it appears that under Fisher's model, the expected fitness change due to the fixation of a new mutation in a population does not depend much on the effective population size. Though mathematically explicable, it is nevertheless biologically surprising since the effective population size of a population is thought to be important in determining the 
fate of the population. One way to understand this is to realize that the expected fitness change can be different from one observed outcome in nature.

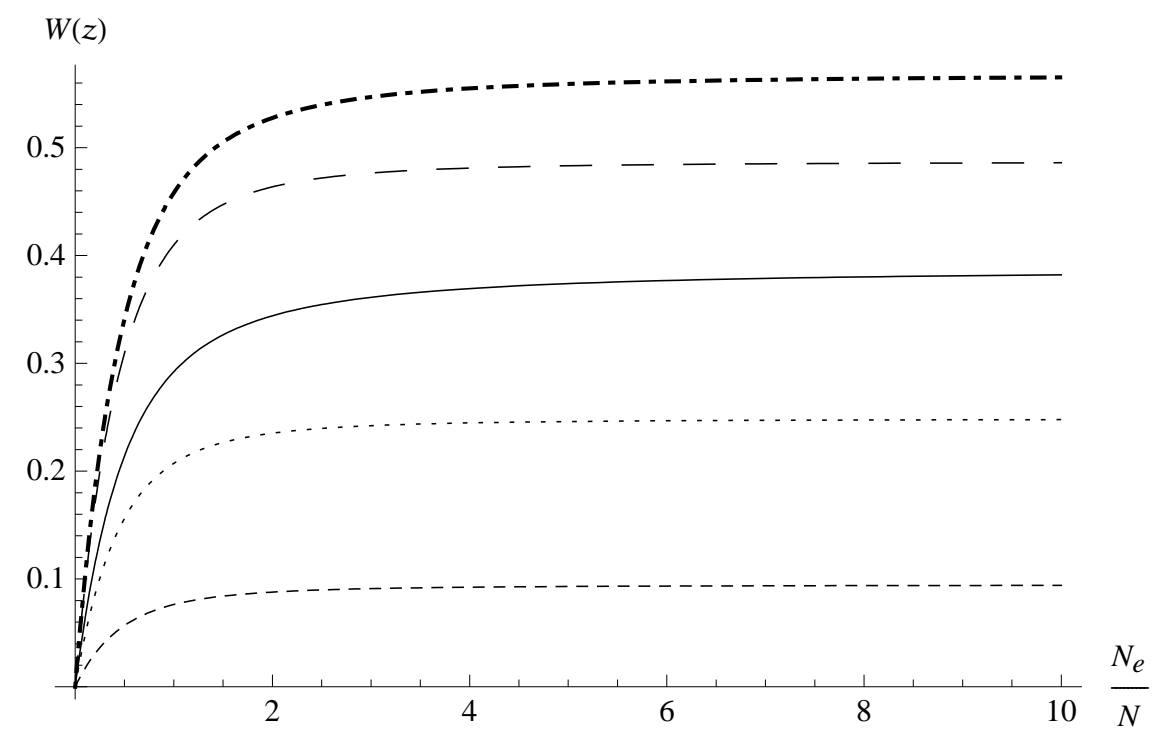

Figure 5: The effect of the ratio $\frac{N_{e}}{N}$ on the expected fitness change of a new mutation with $\beta=1$, $z=3$, and $N_{e}=1000$ for all the curves, but with different $\alpha: \alpha=0.5$ (black), $\alpha=1$ (dotdashed), $\alpha=2$ (long-dashed), $\alpha=5$ (dotted), and $\alpha=10$ (short-dashed). Notice the nonmonotone behavior of $W(z)$ with respect to $\alpha$ for a fixed $\frac{N_{e}}{N}$.

\section{The always positive expected fitness change}

Since populations do go to extinction, one might expect $W(z)$ to be negative for some distribution parameters $\alpha>0, \beta>0$ and initial state $z$. A rigorous proof that $W(z)>0$ for all $\alpha>0$, $\beta>0$, and $z \neq 0$ appears to be difficult, but there is overwhelming computational evidence that this is so. There are several possible explanations for this. One explanation is purely technical. Observe that the fixation probability $u(s)$ is strictly increasing with $u(-\infty)=0, u(0)=1 /(2 N)$, and $u(\infty)=1$. Furthermore, $u(s)$ is hugely skewed in favor of beneficial mutations (fitness effect $s>0)$. For example, with $N_{e}=100, N=1000$,

$$
u(-0.1)=10^{-19}, \quad u(0.1)=0.020, \quad u(-0.5)=10^{-88}, \quad u(0.5)=0.095 .
$$


Thus, in this case, the integrand in (4) is essentially zero for $s=\left(|z|-\left|z^{\prime}\right|\right) /|z|<-0.1$, which is most of the interval $-\infty<z^{\prime}<\infty$, positive for $s>0$, and negative and nonnegligible only for $-0.1<s<0$. Because of the shape of $u(s)$, the positive integral $\int_{0}^{1}(\cdot) d s$ is larger in magnitude than the negative integral $\int_{-0.1}^{0}(\cdot) d s$, giving $W(z)>0$.

Another explanation recalls the definition of $W(z)$ as the expected fitness effect of a mutation from the initial population state $z$. Thus while the expected fitness effect is positive, deleterious mutations can occur and fix in the population, driving the population to extinction with positive probability - this is just not the expected (or average) outcome.

Another explanation is that the model here is not correct. Fitness effects may not be so simply related to mutation distances. The particular definition of fitness effect $s$ used here may be invalid $\left(W(A)=e^{-|z|}\right)$. The choice of the function representing the relationship between mutation lengths and fitness effect can influence the outcome of the model. A previous study used $W(A)=e^{-\sigma|z|^{2}}(\sigma$ is the common nonnegative intensity of selection on all traits) to define the relationship [13]. These two functions are a simplification of nature, where fitness effect of mutation and mutation lengths can have a multitude of different relationships. Additionally, the fixation probability $u(s)$ used here may be incorrect or invalid for the particular definition of $s$ used here. The assumed gamma distribution of mutation lengths $\left|z^{\prime}-z\right|$ may not correspond to nature. While each component of the model here is an accepted model from the literature, a model is only as good as its weakest submodel or assumption.

Nevertheless, under Fisher's geometric adaptation model, with the current assumptions similar to those in the literature (e.g., the assumption of a gamma distribution for the distribution of mutation lengths [9]), results show that the expected fitness change due to the fixation of a mutation is positive. This suggests that if one follows through a population, fixation of mutations over the long term is expected to lead to fitness gains for the population, regardless of the effective population size of the population. Thus, small populations may not necessarily be doomed due to the runaway process of fixation of deleterious mutations. It has been shown that 
incorporating the effect of sexual selection [15] or reverse mutations [7] into theoretical models can greatly reduce the risk of small population extinction. Note that the current results focus on character distribution in one dimension. In reality, there might be many characteristics that interact together to determine the final fitness cost of a mutation [12]. It has been shown that increasing the number of dimensions that contribute to the fitness effect (pleiotropy) of mutations reduces the mutation load of populations and thus the risk of a small population going to extinction [9]. However, all the cited theoretical studies rely on theoretical assumptions, inferred from empirical studies, and understanding of mutational effect on the fitness [13]. An increasing number of studies suggest that one mutation can have different effects on different traits, and mutations themselves can compensate for each other's deleterious effect (compensatory mutations, e.g. $[1,10])$. This poses a challenge for how to model the complex interactions between mutations and their fitness effect (e.g. $[5,14])$. At the same time, because of a dearth of empirical data on the fitness effect of a mutation, further studies should put more emphasis on somehow measuring the fitness effect of a mutation empirically and understanding how the fitness effect of a mutation is determined by the interaction of different genetic components of a population.

The present study focuses on understanding the expected fitness cost of a mutation using the one-dimensional Fisher model, and applies to species with low mutation rates. For species with high mutation rates, multiple mutations may exist at the same time in the population, requiring consideration of the effect of the fixation of multiple mutations. Work has been done to understand the effect of multiple mutations on populations that have high mutation rates (e.g., $[3,11,16])$. Future work will consider the fixation of multiple mutations and also higher dimensions (e.g. $[5,14])$.

Finally, since considerable effort went into deriving the approximation for $W(z)$ based on $s=1-\left|z^{\prime} / z\right|$, why was that approximation not compared to the "true" $W(z)$ ? First, the analytical study was motivated by the question of what, if anything, could be done analytically with $W(z)$ based on $s=e^{|z|-\left|z^{\prime}\right|}-1$. The answer is probably nothing, since the derivation here 
with the simplification $s=1-\left|z^{\prime} / z\right|$ is highly nontrivial, requiring generalized gamma and zeta functions. Second, suppose an analytic form of $W(z)$, in terms of special functions, did exist. It turns out that numerically evaluating these special functions, and infinite series in them, is at least as expensive as directly evaluating numerically the integral in (4). So, embarrassingly (but well known to numerical analysts!), even if one had an analytical form for $W(z)$, it probably would not be used in practice.

\section{ACKNOWLEDGMENTS}

This work was supported in part by NIGMS/NIH Grant 5 R01 GM078989, NSF Grants DMI0355391, CCF-0726763, and IIS-0710945, Department of Energy Grant DE-FG02-06ER25720, and NSF AdvanceVT grant.

\section{References}

[1] J. M. Carlson, Z. L. Brumme, C. M. Rousseau, C. J. Brumme, P. Matthews, C. Kadie, J. I. Mullins, B. D. Walker, P. R. Harrigan, P. J. Goulder, and D. Heckerman. Phylogenetic dependency networks: inferring patterns of CTL escape and codon covariation in HIV-1 Gag. PLoS Comput Biol, 4(11):e1000225, 2008.

[2] J. F. Crow and M. Kimura. An Introduction to Population Genetics Theory. Harper Row, New York, NY, 1970.

[3] M. M. Desai, D. S. Fisher, and A. W. Murray. The speed of evolution and maintenance of variation in asexual populations. Curr Biol, 17(5):385-94, 2007.

[4] R.A. Fisher. Genetical Theory of Natural Selection. The Clarendon Press, Oxford, U.K., 1930.

[5] C. S. Gokhale, Y. Iwasa, M. A. Nowak, and A. Traulsen. The pace of evolution across fitness valleys. J Theor Biol, 259(3):613-20, 2009. 
[6] D. L. Hartl and C. H. Taubes. Compensatory nearly neutral mutations: selection without adaptation. J Theor Biol, 182(3):303-9, 1996.

[7] R. Lande. Risk of population extinction from fixation of deleterious and reverse mutations. Genetica, 102-103(1-6):21-7, 1998.

[8] H. A. Orr. The distribution of fitness effects among beneficial mutations in Fisher's geometric model of adaptation. J Theor Biol, 238(2):279-85, 2006.

[9] A. Poon and S. P. Otto. Compensating for our load of mutations: freezing the meltdown of small populations. Evolution Int J Org Evolution, 54(5):1467-79, 2000.

[10] A. F. Poon, F. I. Lewis, S. L. Pond, and S. D. Frost. Evolutionary interactions between N-linked glycosylation sites in the HIV-1 envelope. PLoS Comput Biol, 3(1):e11, 2007.

[11] I. M. Rouzine, E. Brunet, and C. O. Wilke. The traveling-wave approach to asexual evolution: Muller's ratchet and speed of adaptation. Theor Popul Biol, 73(1):24-46, 2008.

[12] G.P. Wagner, ed. The character concept in evolutionary biology. Academic Press, San Diego, CA, 2001.

[13] D. Waxman and J. J. Welch. Fisher's microscope and Haldane's ellipse. Am Nat, 166(4):447$57,2005$.

[14] D. B. Weissman, M. M. Desai, D. S. Fisher, and M. W. Feldman. The rate at which asexual populations cross fitness valleys. Theor Popul Biol, 75(4):286-300, 2009.

[15] M. C. Whitlock. Fixation of new alleles and the extinction of small populations: drift load, beneficial alleles, and sexual selection. Evolution Int J Org Evolution, 54(6):1855-61, 2000.

[16] C. Zeyl. Evolutionary genetics: a piggyback ride to adaptation and diversity. Curr Biol, 17(9):R333-5, 2007. 
[17] L. Zhang and L. T. Watson. Note on the computation of critical effective population sizes. J Comput Biol, 14(7):950-60, 2007.

[18] L. Zhang and L.T. Watson. Analysis of the fitness effect of compensatory mutations. HFSP $J, 3(1): 47-54,2009$.

\section{Appendix: Derivation of $W_{1}(z)$}

A closed form expression for each of $D_{1}, D_{2}, \ldots, D_{6}$ will be derived in turn. One would like to write

$$
\begin{aligned}
D_{1} & =\int_{-\infty}^{0} \frac{\left(z-z^{\prime}\right)^{\alpha-1} \beta^{\alpha} e^{-\beta\left(z-z^{\prime}\right)}}{\Gamma(\alpha)} \frac{1-e^{-2\left(1+\frac{z^{\prime}}{z}\right)}}{1-e^{-4 N_{e}\left(1+\frac{z^{\prime}}{z}\right)}} d z^{\prime} \\
& =\frac{\beta^{\alpha}}{\Gamma(\alpha)}[\underbrace{\int_{-\infty}^{0} \frac{\left(z-z^{\prime}\right)^{\alpha-1} e^{-\beta\left(z-z^{\prime}\right)}}{1-e^{-4 N_{e}\left(1+\frac{z^{\prime}}{z}\right)}} d z^{\prime}}_{A_{0}}-\underbrace{\int_{-\infty}^{0} \frac{\left(z-z^{\prime}\right) e^{-\beta\left(z-z^{\prime}\right)} e^{-2\left(1+\frac{z^{\prime}}{z}\right)}}{1-e^{-4 N_{e}\left(1+\frac{z^{\prime}}{z}\right)}} d z^{\prime}}_{B_{0}}],
\end{aligned}
$$

however, this is mathematically invalid since the integrals $A_{0}$ and $B_{0}$ do not exist; for instance, $A_{0}$ contains the improper integral

$$
\int_{-z-\epsilon}^{-z} \frac{1}{z+z^{\prime}} d z^{\prime}=-\infty
$$

for small $\epsilon>0$. (Near $z^{\prime}=-z$, the numerator of $A_{0}$ is integrable and positive, and the denominator expands to $\frac{4 N_{e}}{z}\left(z+z^{\prime}\right)+o\left(z+z^{\prime}\right)$.) The technical difficulty is that while the fixation probability $u(s)$ is analytic for all $s$, it can be split apart as

$$
u(s)=\frac{1-e^{-2 s}}{1-e^{-4 N_{e} s}}=\frac{1}{1-e^{-4 N_{e} s}}-\frac{e^{-2 s}}{1-e^{-4 N_{e} s}}
$$

only for $s \neq 0$. Thus $D_{1}$ must be written as

$$
D_{1}=\int_{-\infty}^{0}=\int_{-\infty}^{-z-\epsilon}+\int_{-z-\epsilon}^{-z+\epsilon}+\int_{-z+\epsilon}^{0}
$$


for some $0<\epsilon \ll 1$. $u(s)$ can be split apart in the first and last integrals, but not the middle one, which approaches 0 as $\epsilon \rightarrow 0$. Thus, $D_{1}$ must be decomposed as

$$
D_{1}=\frac{\beta^{\alpha}}{\Gamma(\alpha)}\left[A_{0,1}+B_{0,1}+\int_{-z-\epsilon}^{-z+\epsilon}+A_{0,2}+B_{0,2}\right]
$$

where the small integral $\int_{-z-\epsilon}^{-z+\epsilon}$ is either dropped or approximated numerically, and the remaining terms are given exactly by

$$
\begin{aligned}
A_{0,1} & =\int_{-\infty}^{-z-\epsilon} \frac{\left(z-z^{\prime}\right)^{\alpha-1} e^{-\beta\left(z-z^{\prime}\right)} e^{4 N_{e}\left(1+\frac{z^{\prime}}{z}\right)}}{e^{4 N_{e}\left(1+\frac{z^{\prime}}{z}\right)}-1} d z^{\prime} \\
& =-\int_{-\infty}^{-z-\epsilon} \frac{\left(z-z^{\prime}\right)^{\alpha-1} e^{-\beta z+\beta z^{\prime}+4 N_{e}+4 N_{e} \frac{z^{\prime}}{z}}}{1-e^{4 N_{e}\left(1+\frac{z^{\prime}}{z}\right)}} d z^{\prime} \\
& =-z^{\alpha-1} \int_{-\infty}^{-z-\epsilon}\left(1-\frac{z^{\prime}}{z}\right)^{\alpha-1} e^{-\beta z+\beta z^{\prime}+4 N_{e}+4 N_{e} \frac{z^{\prime}}{z}} \sum_{t=0}^{\infty} e^{4 N_{e} t\left(1+\frac{z^{\prime}}{z}\right)} d z^{\prime} \\
& =-z^{\alpha-1} \sum_{t=0}^{\infty} \int_{-\infty}^{-z-\epsilon}\left(1-\frac{z^{\prime}}{z}\right)^{\alpha-1} e^{-\beta z+\beta z^{\prime}+4 N_{e}+4 N_{e} \frac{z^{\prime}}{z}+4 N_{e} t+4 N_{e} t \frac{z^{\prime}}{z}} d z^{\prime} \\
& =-\sum_{t=0}^{\infty} e^{8 N_{e}+8 N_{e} t} z^{\alpha-1} \int_{-\infty}^{-z-\epsilon}\left(1-\frac{z^{\prime}}{z}\right)^{\alpha-1} e^{-\left(4 N_{e}+4 N_{e} t+\beta z\right)\left(1-\frac{z^{\prime}}{z}\right)} d z^{\prime} \\
& =-\sum_{t=0}^{\infty} z^{\alpha} e^{8 N_{e}+8 N_{e} t} \int_{2+\epsilon / z}^{\infty} s^{\alpha-1} e^{-\left(4 N_{e}+4 N_{e} t+\beta z\right) s} d s \quad\left(\text { with } s=1-\frac{z^{\prime}}{z}\right) \\
& =-\sum_{t=0}^{\infty} \frac{z^{\alpha} e^{8 N_{e}+8 N_{e} t} \gamma\left(\alpha,(2+\epsilon / z)\left(4 N_{e}+4 N_{e} t+\beta z\right)\right)}{\left(4 N_{e}+4 N_{e} t+\beta z\right)^{\alpha}} \\
& \left(\text { with } x=\left(4 N_{e}+4 N_{e} t+\beta z\right) s\right),
\end{aligned}
$$

$$
\begin{aligned}
A_{0,2} & =\int_{-z+\epsilon}^{0}\left(z-z^{\prime}\right)^{\alpha-1} e^{-\beta\left(z-z^{\prime}\right)} \sum_{t=0}^{\infty} e^{-4 N_{e} t\left(1+\frac{z^{\prime}}{z}\right)} d z^{\prime} \\
& =\sum_{t=0}^{\infty} z^{\alpha-1} \int_{-z+\epsilon}^{0}\left(1-\frac{z^{\prime}}{z}\right)^{\alpha-1} e^{-\left(1-\frac{z^{\prime}}{z}\right)\left(-4 N_{e} t+\beta z\right)-8 N_{e} t} d z^{\prime} \\
& =\sum_{t=0}^{\infty} z^{\alpha} e^{-8 N_{e} t} \int_{1}^{2-\epsilon / z} s^{\alpha-1} e^{-s\left(\beta z-4 N_{e} t\right)} d s \quad\left(\text { with } s=1-\frac{z^{\prime}}{z}\right) \\
& =\sum_{t=0}^{\left\lfloor\frac{\beta z}{4 N_{e}}\right\rfloor} \frac{z^{\alpha} e^{-8 N_{e} t}}{\left(\beta z-4 N_{e} t\right)^{\alpha}} \hat{\gamma}\left(\alpha, \beta z-4 N_{e} t,(2-\epsilon / z)\left(\beta z-4 N_{e} t\right)\right)
\end{aligned}
$$




$$
\begin{aligned}
& +\sum_{t=\left\lfloor\frac{\beta z}{4 N_{e}}\right\rfloor+1}^{\infty} z^{\alpha} e^{-8 N_{e} t} \int_{1}^{2-\epsilon / z} s^{\alpha-1} e^{-s\left(\beta z-4 N_{e} t\right)} d s \\
= & \sum_{t=0}^{\left\lfloor\frac{\beta z}{4 N_{e}}\right\rfloor} \frac{z^{\alpha} e^{-8 N_{e} t} \hat{\gamma}\left(\alpha, \beta z-4 N_{e} t,(2-\epsilon / z)\left(\beta z-4 N_{e} t\right)\right)}{\left(\beta z-4 N_{e} t\right)^{\alpha}} \\
& +\sum_{t=\left\lfloor\frac{\beta z}{4 N_{e}}\right\rfloor+1}^{\infty} z^{\alpha} e^{-8 N_{e} t} \sum_{k=0}^{\infty} \frac{\left((2-\epsilon / z)^{k+\alpha}-1\right)\left(4 N_{e} t-\beta z\right)^{k}}{k !(k+\alpha)} \\
B_{0,1}=- & \int_{-\infty}^{-z-\epsilon}\left(z-z^{\prime}\right)^{\alpha-1} e^{-\beta z+\beta z^{\prime}-2-2 \frac{z^{\prime}}{z}+4 N_{e}+4 N_{e} \frac{z^{\prime}}{z}} \sum_{t=0}^{\infty} e^{4 N_{e} t\left(1+\frac{z^{\prime}}{z}\right)} \\
=- & z^{\alpha-1} \sum_{t=0}^{\infty} e^{8 N_{e}+8 N_{e} t-4} \int_{-\infty}^{-z-\epsilon}\left(1-\frac{z^{\prime}}{z}\right)^{\alpha-1} e^{-\left(1-\frac{z^{\prime}}{z}\right)\left(4 N_{e}+4 N_{e} t-2+\beta z\right)} d z^{\prime} \\
=- & \sum_{t=0}^{\infty} \frac{z^{\alpha} e^{8 N_{e}+8 N_{e} t-4} \gamma\left(\alpha,(2+\epsilon / z)\left(4 N_{e}+4 N_{e} t-2+\beta z\right)\right)}{\left(4 N_{e}+4 N_{e} t-2+\beta z\right)^{\alpha}}
\end{aligned}
$$

and

$$
\begin{aligned}
B_{0,2}= & z^{\alpha-1} \sum_{t=0}^{\infty} \int_{-z+\epsilon}^{0}\left(1-\frac{z^{\prime}}{z}\right)^{\alpha-1} e^{-\left(1-\frac{z^{\prime}}{z}\right)\left(\beta z-2-4 N_{e} t\right)-4-8 N_{e} t} d z^{\prime} \\
= & \sum_{t=0}^{\left\lfloor\frac{\beta z+2}{4 N_{e}}\right\rfloor} \frac{z^{\alpha} e^{-4-8 N_{e} t} \hat{\gamma}\left(\alpha, \beta z-2-4 N_{e} t, 2\left(\beta z-2-4 N_{e} t\right)\right)}{\left(\beta z-2-4 N_{e} t\right)^{\alpha}} \\
& +\sum_{t=\left\lfloor\frac{\beta z+2}{4 N_{e}}\right\rfloor+1}^{\infty} z^{\alpha} e^{-4-8 N_{e} t} \sum_{k=0}^{\infty} \frac{\left((2-\epsilon / z)^{k+\alpha}-1\right)\left(2+4 N_{e} t-\beta z\right)^{k}}{k !(k+\alpha)}
\end{aligned}
$$

$D_{2}$ and $D_{3}$ are somewhat easier to compute than $D_{1}$. As with $D_{1}$, care must be taken to avoid infinite integrals. Converting the denominator of $u(s)$ to a geometric series yields

$$
\begin{aligned}
D_{2} & -\int_{z-\epsilon}^{z} f\left(z^{\prime}\right) u(s) d z^{\prime} \\
& =\int_{0}^{z-\epsilon} \frac{\beta^{\alpha}\left(z-z^{\prime}\right)^{\alpha-1} e^{-\beta\left(z-z^{\prime}\right)}}{\Gamma(\alpha)}\left(1-e^{-2\left(1-\frac{z^{\prime}}{z}\right)}\right) \sum_{t=0}^{\infty} e^{-4 N_{e} t\left(1-\frac{z^{\prime}}{z}\right)} d z^{\prime} \\
& =\frac{z^{\alpha-1} \beta^{\alpha}}{\Gamma(\alpha)} \sum_{t=0}^{\infty} \int_{0}^{z-\epsilon}\left(1-\frac{z^{\prime}}{z}\right)^{\alpha-1} e^{-\beta z\left(1-\frac{z^{\prime}}{z}\right)}\left(1-e^{-2\left(1-\frac{z^{\prime}}{z}\right)}\right) e^{-4 N_{e} t\left(1-\frac{z^{\prime}}{z}\right)} d z^{\prime}
\end{aligned}
$$




$$
\begin{gathered}
=\frac{z^{\alpha-1} \beta^{\alpha}}{\Gamma(\alpha)} \sum_{t=0}^{\infty}[\underbrace{\int_{0}^{z-\epsilon}\left(1-\frac{z^{\prime}}{z}\right)^{\alpha-1} e^{-\beta z\left(1-\frac{z^{\prime}}{z}\right)-4 N_{e} t\left(1-\frac{z^{\prime}}{z}\right)} d z^{\prime}}_{D_{2,1}} \\
-\underbrace{\int_{0}^{z-\epsilon}\left(1-\frac{z^{\prime}}{z}\right)^{\alpha-1} e^{-\beta z\left(1-\frac{z^{\prime}}{z}\right)-2\left(1-\frac{z^{\prime}}{z}\right)-4 N_{e} t\left(1-\frac{z^{\prime}}{z}\right)} d z^{\prime}}_{D_{2,2}}] .
\end{gathered}
$$

Now working on the integrals $D_{2,1}$ and $D_{2,2}$,

$$
\begin{aligned}
D_{2,1} & =\int_{0}^{z-\epsilon}\left(1-\frac{z^{\prime}}{z}\right)^{\alpha-1} e^{-\left(\beta z+4 N_{e} t\right)\left(1-\frac{z^{\prime}}{z}\right)} d z^{\prime} \\
& \left.=z \int_{\epsilon / z}^{1} s^{\alpha-1} e^{-\left(\beta z+4 N_{e} t\right) s} d s \quad \text { (with } s=1-\frac{z^{\prime}}{z}\right) \\
& =z\left(\beta z+4 N_{e} t\right)^{-\alpha} \int_{\epsilon / z}^{1}\left[\left(\beta z+4 N_{e} t\right) s\right]^{\alpha-1} e^{-\left(\beta z+4 N_{e} t\right) s} d\left(\beta z+4 N_{e} t\right) s \\
& \left.=\frac{z}{\left(\beta z+4 N_{e} t\right)^{\alpha}} \int_{\epsilon / z\left(\beta z+4 N_{e} t\right)}^{\beta z+4 N_{e} t} x^{\alpha-1} e^{-x} d x \quad \quad \text { (with } x=\left(\beta z+4 N_{e} t\right) s\right) \\
& =\frac{z \hat{\gamma}\left(\alpha, \epsilon / z\left(\beta z+4 N_{e} t\right), \beta z+4 N_{e} t\right)}{\left(\beta z+4 N_{e} t\right)^{\alpha}},
\end{aligned}
$$

and

$$
\begin{aligned}
D_{2,2} & =\int_{0}^{z-\epsilon}\left(1-\frac{z^{\prime}}{z}\right) e^{-\beta z\left(1-\frac{z^{\prime}}{z}\right)-2\left(1-\frac{z^{\prime}}{z}\right)-4 N_{e} t\left(1-\frac{z^{\prime}}{z}\right)} d z^{\prime} \\
& =z \int_{\epsilon / z}^{1} s^{\alpha-1} e^{-\beta z s-2 s-4 N_{e} t s} d s \quad\left(\text { with } s=1-\frac{z^{\prime}}{z}\right) \\
& =\frac{z \hat{\gamma}\left(\alpha,(\epsilon / z)\left(\beta z+4 N_{e} t+2\right), \beta z+4 N_{e} t+2\right)}{\left(\beta z+4 N_{e} t+2\right)^{\alpha}} .
\end{aligned}
$$

Combining the expressions for the integrals $D_{2,1}$ and $D_{2,2}$ yields

$$
\begin{aligned}
D_{2}- & \int_{z-\epsilon}^{z} f\left(z^{\prime}\right) u(s) d z^{\prime} \\
= & \frac{z^{\alpha-1} \beta^{\alpha}}{\Gamma(\alpha)} \sum_{t=0}^{\infty}\left[\frac{z \hat{\gamma}\left(\alpha, \epsilon / z\left(\beta z+4 N_{e} t\right), \beta z+4 N_{e} t\right)}{\left(\beta z+4 N_{e} t\right)^{\alpha}}\right. \\
& \left.-\frac{z \hat{\gamma}\left(\alpha,(\epsilon / z)\left(\beta z+4 N_{e} t+2\right), \beta z+4 N_{e} t+2\right)}{\left(\beta z+4 N_{e} t+2\right)^{\alpha}}\right]
\end{aligned}
$$




$$
\begin{gathered}
=\frac{(\beta z)^{\alpha}}{\left(4 N_{e}\right)^{\alpha} \Gamma(\alpha)} \sum_{t=0}^{\infty}\left[\frac{\hat{\gamma}\left(\alpha, \epsilon / z\left(\beta z+4 N_{e} t\right), \beta z+4 N_{e} t\right)}{\left(t+\frac{\beta z}{4 N_{e}}\right)^{\alpha}}\right. \\
\left.-\frac{\hat{\gamma}\left(\alpha,(\epsilon / z)\left(\beta z+4 N_{e} t+2\right), \beta z+4 N_{e} t+2\right)}{\left(t+\frac{\beta z+2}{4 N_{e}}\right)^{\alpha}}\right] .
\end{gathered}
$$

Using now the substitutions $s=\frac{z^{\prime}}{z}-1$ and $x=\left(\beta z+4 N_{e}+4 N_{e} t-2\right) s$,

$$
\begin{aligned}
D_{3}- & \int_{z}^{z+\epsilon} f\left(z^{\prime}\right) u(s) d z^{\prime} \\
= & \frac{\beta^{\alpha}}{\Gamma(\alpha)} \int_{z+\epsilon}^{\infty}\left(z^{\prime}-z\right)^{\alpha-1} e^{-\beta\left(z^{\prime}-z\right)} \frac{e^{4 N_{e}\left(1-\frac{z^{\prime}}{z}\right)}\left(e^{-2\left(1-\frac{z^{\prime}}{z}\right)}-1\right)}{1-e^{4 N_{e}\left(1-\frac{z^{\prime}}{z}\right)}} d z^{\prime} \\
= & \frac{\beta^{\alpha}}{\Gamma(\alpha)} \sum_{t=0}^{\infty} \int_{z+\epsilon}^{\infty}\left(z^{\prime}-z\right)^{\alpha-1} e^{-\beta\left(z^{\prime}-z\right)+4 N_{e}\left(1-\frac{z^{\prime}}{z}\right)+4 N_{e} t\left(1-\frac{z^{\prime}}{z}\right)}\left(e^{-2\left(1-\frac{z^{\prime}}{z}\right)}-1\right) d z^{\prime} \\
= & \frac{\beta^{\alpha}}{\Gamma(\alpha)} \sum_{t=0}^{\infty}\left(\frac{z^{\alpha} \gamma\left(\alpha, \epsilon / z\left(\beta z+4 N_{e}+4 N_{e} t-2\right)\right)}{\left(\beta z+4 N_{e}+4 N_{e} t-2\right)^{\alpha}}-\frac{z^{\alpha} \gamma\left(\alpha, \epsilon / z\left(\beta z+4 N_{e}+4 N_{e} t\right)\right)}{\left(\beta z+4 N_{e}+4 N_{e} t\right)^{\alpha}}\right) \\
= & \left(\frac{\beta z}{4 N_{e}}\right)^{\alpha} \frac{1}{\Gamma(\alpha)} \sum_{t=0}^{\infty}\left[\frac{\gamma\left(\alpha, \epsilon / z\left(\beta z+4 N_{e}+4 N_{e} t-2\right)\right)}{\left(t+\frac{\beta z+4 N_{e}-2}{4 N_{e}}\right)^{\alpha}}\right. \\
& \left.-\frac{\gamma\left(\alpha, \epsilon / z\left(\beta z+4 N_{e}+4 N_{e} t\right)\right)}{\left(t+\frac{\beta z+4 N_{e}}{4 N_{e}}\right)^{\alpha}}\right] .
\end{aligned}
$$

This completes the analysis of $W_{1}(z)$ for $z>0$. Now turn to $z<0$ where $W_{1}(z)=D_{4}+D_{5}+D_{6}$. As earlier, it is necessary to write

$$
D_{4}=\int_{0}^{\infty}=\int_{0}^{-z-\epsilon}+\int_{-z-\epsilon}^{-z+\epsilon}+\int_{-z+\epsilon}^{\infty}=D_{4,1}+\int_{-z-\epsilon}^{-z+\epsilon}+D_{4,2}
$$

where

$$
\begin{aligned}
D_{4,1}= & \frac{\beta^{\alpha}}{\Gamma(x)} \int_{0}^{-z-\epsilon} \frac{\left(z^{\prime}-z\right)^{\alpha-1} e^{-\beta\left(z^{\prime}-z\right)}\left(1-e^{-2\left(1+\frac{z^{\prime}}{z}\right)}\right)}{1-e^{-4 N_{e}\left(1+\frac{z^{\prime}}{z}\right)}} d z^{\prime} \\
= & \sum_{t=0}^{\left\lfloor\frac{-\beta z}{4 N_{e}}\right\rfloor} \frac{(-z)^{\alpha} e^{-8 N_{e} t} \hat{\gamma}\left(\alpha,\left(-4 N_{e} t-\beta z\right),(2+\epsilon / z)\left(-4 N_{e} t-\beta z\right)\right)}{\left(-4 N_{e} t-\beta z\right)^{\alpha}} \\
& +\sum_{t=\left\lfloor\frac{-\beta z}{4 N_{e}}\right\rfloor+1}^{\infty}(-z)^{\alpha} e^{-8 N_{e} t} \sum_{k=0}^{\infty} \frac{\left(4 N_{e} t+\beta z\right)^{k}\left((2+\epsilon / z)^{k+\alpha}-1\right)}{k !(k+\alpha)} \\
& -\sum_{t=0}^{\left\lfloor\frac{\beta z+2}{\left.-4 N_{e}\right\rfloor}\right.} \frac{(-z)^{\alpha} e^{-4-8 N_{e} t} \hat{\gamma}\left(\alpha,\left(-2-4 N_{e} t-\beta z\right),(2+\epsilon / z)\left(-2-4 N_{e} t-\beta z\right)\right)}{\left(-2-4 N_{e} t-\beta z\right)^{\alpha}}
\end{aligned}
$$




$$
\begin{aligned}
& -\sum_{t=\left\lfloor\frac{\beta z+2}{\left.-4 N_{e}\right\rfloor+1}\right.}^{\infty}(-z)^{\alpha} e^{-4-8 N_{e} t} \sum_{k=0}^{\infty} \frac{\left(2+4 N_{e} t+\beta z\right)^{k}\left((2+\epsilon / z)^{k+\alpha}-1\right)}{k !(k+\alpha)}, \\
D_{4,2}= & \frac{\beta^{\alpha}}{\Gamma(x)} \int_{-z+\epsilon}^{\infty} \frac{\left(z^{\prime}-z\right)^{\alpha-1} e^{-\beta\left(z^{\prime}-z\right)}\left(1-e^{-2\left(1+\frac{z^{\prime}}{z}\right)}\right)}{1-e^{-4 N_{e}\left(1+\frac{z^{\prime}}{z}\right)}} d z^{\prime} \\
= & \sum_{t=0}^{\infty}(-z)^{\alpha} e^{8 N_{e}+8 N_{e} t}\left[\frac{e^{-4} \gamma\left(\alpha,(2-\epsilon / z)\left(4 N_{e}+4 N_{e} t-\beta z-2\right)\right)}{\left(4 N_{e}+4 N_{e} t-\beta z-2\right)^{\alpha}}\right. \\
& \left.-\frac{\gamma\left(\alpha,(2-\epsilon / z)\left(4 N_{e}+4 N_{e} t-\beta z\right)\right)}{\left(4 N_{e}+4 N_{e} t-\beta z\right)^{\alpha}}\right] .
\end{aligned}
$$

Writing $D_{5}-\int_{z-\epsilon}^{z} f\left(z^{\prime}\right) u(s) d z^{\prime}=D_{5,1}+D_{5,2}$ and using the variable substitution $s=\frac{z^{\prime}}{z}-1$ leads to

$$
\begin{aligned}
D_{5,1} & =\frac{-\beta^{\alpha}}{\Gamma(\alpha)} \sum_{t=0}^{\infty} \int_{-\infty}^{z-\epsilon}\left(z-z^{\prime}\right)^{\alpha-1} e^{-\beta z+\beta z^{\prime}+4 N_{e}\left(1-\frac{z^{\prime}}{z}\right)+4 N_{e} t\left(1-\frac{z^{\prime}}{z}\right)} d z^{\prime}, \\
& =\frac{-(-\beta z)^{\alpha}}{\Gamma(\alpha)} \sum_{t=0}^{\infty} \int_{-\epsilon / z}^{\infty} s^{\alpha-1} e^{-s\left(4 N_{e}+4 N_{e} t-\beta z\right)} d s \\
& =\frac{-(-\beta z)^{\alpha}}{\Gamma(\alpha)} \sum_{t=0}^{\infty} \frac{\gamma\left(\alpha,-\epsilon / z\left(4 N_{e}+4 N_{e} t-\beta z\right)\right)}{\left(4 N_{e}+4 N_{e} t-\beta z\right)^{\alpha}}, \\
D_{5,2} & =\frac{\beta^{\alpha}}{\Gamma(\alpha)} \sum_{t=0}^{\infty} \int_{-\infty}^{z-\epsilon}\left(z-z^{\prime}\right)^{\alpha-1} e^{-\beta z+\beta z^{\prime}-2\left(1-\frac{z^{\prime}}{z}\right)+4 N_{e}\left(1-\frac{z^{\prime}}{z}\right)+4 N_{e} t\left(1-\frac{z^{\prime}}{z}\right)} d z^{\prime} \\
& =\frac{(-\beta z)^{\alpha}}{\Gamma(\alpha)} \sum_{t=0}^{\infty} \frac{\gamma\left(\alpha,-\epsilon / z\left(4 N_{e}+4 N_{e} t-\beta z-2\right)\right)}{\left(4 N_{e}+4 N_{e} t-\beta z-2\right)^{\alpha}} .
\end{aligned}
$$

Similarly,

$$
\begin{aligned}
D_{6}-\int_{z}^{z+\epsilon} f\left(z^{\prime}\right) u(s) d z^{\prime} & =\frac{(-\beta z)^{\alpha}}{\Gamma(\alpha)}\left[\sum_{t=0}^{\infty}\left(\frac{\hat{\gamma}\left(\alpha,-\epsilon / z\left(4 N_{e} t-\beta z\right), 4 N_{e} t-\beta z\right)}{\left(4 N_{e} t-\beta z\right)^{\alpha}}\right)\right. \\
& \left.-\sum_{t=0}^{\infty}\left(\frac{\hat{\gamma}\left(\alpha,-\epsilon / z\left(4 N_{e} t+2-\beta z\right), 4 N_{e} t+2-\beta z\right)}{\left(4 N_{e} t+2-\beta z\right)^{\alpha}}\right)\right]
\end{aligned}
$$

This completes the calculation of $W_{1}(z)$ for all $z \neq 0$ (it is assumed that the current population state is not at its optimum $z=0$ ). 